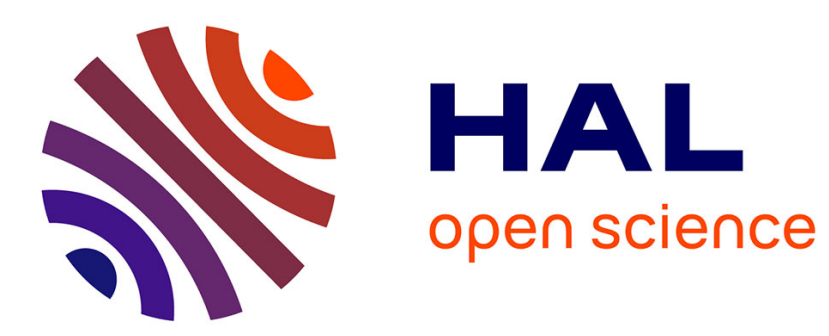

\title{
L'histoire des débordements industriels à l'origine de conflits autour de l'environnement
}

\author{
Michel Letté
}

\section{To cite this version:}

Michel Letté. L'histoire des débordements industriels à l'origine de conflits autour de l'environnement. Annales des mines - Série Responsabilité et environnement, 2011, 62, pp.43-50. 10.3917/re.062.0043 . halshs-03255558

\author{
HAL Id: halshs-03255558 \\ https://shs.hal.science/halshs-03255558
}

Submitted on 9 Jun 2021

HAL is a multi-disciplinary open access archive for the deposit and dissemination of scientific research documents, whether they are published or not. The documents may come from teaching and research institutions in France or abroad, or from public or private research centers.
L'archive ouverte pluridisciplinaire HAL, est destinée au dépôt et à la diffusion de documents scientifiques de niveau recherche, publiés ou non, émanant des établissements d'enseignement et de recherche français ou étrangers, des laboratoires publics ou privés. 


\section{L'histoire des débordements industriels à l'origine de conflits autour de l'environnement}

Michel Letté

Paru dans Annales des Mines - Responsabilité et environnement 2011/2 ( $\left.N^{\circ} 62\right)$, pages 43 à 50 .

Le débordement industriel est ce qui, provenant d'un dispositif de production, investit un environnement donné et suscite la protestation des riverains. En tant que motif récurrent du conflit environnemental, le débordement industriel permet de restituer l'histoire de la contestation environnementale, laquelle recouvre un processus de nature profondément sociale. Un conflit environnemental se conçoit comme une négociation permanente sur les usages spécifiques d'un territoire entre des acteurs (les industriels et les riverains) justifiant d'intérêts contradictoires.

"Industrial overflow" refers to whatever flows from a manufacturing plant over into the environment and sets off complaints from neighbors. This recurrent theme in conflicts related to the environment can serve as a guideline for the history of the deeply social process of environmental protest. An environmental conflict is to be seen as an ongoing negotiation about using land between manufacturers and residents with contradictory interests.

Plan

Du changement technique au changement climatique

Qu'est-ce qu'un conflit environnemental ?

L'historicisation du conflit environnemental

Quel environnement?

Une histoire pragmatique du conflit environnemental

Un motif récurrent de conflit environnemental : le débordement

Quelques exemples de débordements industriels (au XIXe siècle)

Que recouvre le terme de " débordement » ?

Le débordement : un dispositif centré et distribué

Les territoires du débordement

La négociation par le conflit environnemental 
La protestation des populations confrontées aux débordements d'une activité industrielle est pour les pouvoirs publics une préoccupation dont l'histoire, sur le temps long, a fait l'objet, depuis quelques années, de nombreux travaux ${ }^{1}$. L'intervention publique, qui cherche à réguler le comportement des agents économiques et sociaux, y est généralement étudiée pour elle-même. Une des perspectives affichée est d'interroger ce qui a conduit, au $\mathrm{XX}^{\mathrm{e}}$ siècle, à l'émergence de l'environnement en tant que catégorie de la pensée et de l'action tant chez les autorités publiques que parmi les publics concernés ${ }^{2}$.

Toutefois, la plupart de ces études porte sur la période qui débute avec les années $1970^{3}$. Cela suggère que la contestation environnementale ne s'est

1 Thomas Le Roux, Le laboratoire des pollutions industrielles. Paris, 1770-1830, Paris, Albin Michel, 2011; Geneviève Massard-Guilbaud, Histoire de la pollution industrielle. France, 1789-1914, Paris, Editions de l'Ehess, 2010, 404 p., et « Les citadins auvergnats face aux nuisances industrielles, 1810-1914», Recherches contemporaines, 4, 1997, pp. 5-48 ; Jean-Baptiste Fressoz, « The Gas Lighting Controversy : Technological Risk, Expertise, and Regulation in Nineteenth-Century Paris and London ", Journal of Urban History, 33, 2007, pp. 729-755 ; Corinne Beck, Yves Luginbühl et Tatiana Muxart (dir.), Temps et espaces des crises de l'environnement, Editions Quae, 2006, 411 p. ; Estelle Baret-Bourgoin, La ville industrielle et ses poisons: Les mutations des sensibilités aux nuisances et pollutions industrielles à Grenoble, 1810-1914, Grenoble, Presses Universitaires de Grenoble, 2005, 427 p. ; Christoph Bernardt et Geneviève Massard-Guilbaud (dir.), Le démon moderne. La pollution dans les sociétés urbaines et industrielles d'Europe, Clermont-Ferrand, Presses Universitaires Blaise-Pascal, 2002, 464 p.; Pierre-Claude Reynard, «Public order and privilege. Eighteen-century French roots of environmental regulation », Technology and Culture, 43, 2002, pp. 1-48; Pierre-François Claustre, « Une ville saisie par l'industrie : nuisances industrielles et action municipale à Argenteuil (1820-1940)», Recherches contemporaines, 3, 1995-1996, pp. 91-119 ; Gérald Lachaud « Les établissements insalubres de Saint-Étienne au XIX ${ }^{\mathrm{e}}$ siècle » dans Bulletin du Centre Pierre Léon d'histoire économique et sociale, 1994, 1, pp. 3-12 ; Olivier Faure, "L'industrie et "l'environnement" à Lyon au $\mathrm{XIX}^{\mathrm{e}}$ siècle », Recueil d'études offert à Gabriel Désert, 1992, pp. 299-311; Alain Corbin, « L'opinion et la politique face aux nuisances industrielles dans la ville préhausmannienne », Histoire, Economie et Société, 2, 1983, pp. 111-118.

2 Florian Charvolin, L'invention de l'environnement en France: chroniques anthropologiques d'une institutionnalisation, Paris, Éditions de La Découverte, 2003, 133 p.; Yannick Rumpala, Régulation publique et environnement: questions écologiques, réponses économiques, Paris, L'Harmattan, 2003, 373 p. ; Joseph Szarka, The shaping of environmental policy in France, New York, Berghahn Books, 2002, 250 p. ; Marie Cornu et Jérome Fromageau (dir.), Genèse du droit de l'environnement : Droit des espaces naturels et des pollutions, Paris, L'Harmattan, 2001, 282 p. et Genèse du droit de l'environnement : Fondements et enjeux internationaux, 231 p.; Bernard Kalaora, Au-delà de la nature, l'environnement : l'observation sociale de l'environnement, Paris, L'Harmattan, 1998, 199 p. ; Florence Rudolph, L'environnement, une construction sociale. Pratiques et discours sur l'environnement en Allemagne et en France, Presses Universitaires de Strasbourg, 1998, 184 p. ; Pierre Lascoumes, L'Éco-pouvoir. L'environnement entre nature et politique, Paris, La Découverte, 1994, 320 p., et Pierre Lascoumes (dir.), Instituer l'environnement. Vingt-cinq ans d'administration de l'environnement, Paris, L'Harmattan, 1999, 233 p. ; Marie-Claire Robic, Du milieu à l'environnement : pratiques et représentations du rapport homme/nature depuis la Renaissance, Economica, 1992, 343 p.

${ }^{3}$ Bruno Charlier, La défense de l'environnement : entre espace et territoire. Géographie des conflits environnementaux depuis 1974, Thèse de l'Université de Pau, 2 vol., 1999, 750 p. ; Olivier Filleule, «France» dans Chris Rootes (dir.), Environmental Protest in Western Europe, Oxford University Press, 2003, pp. 59-79 ; Graeme Hayes, Environmental protest and the State in France, Palgrave Macmillan, 2002, 246 p.; Anne Guérin-Henri et Dominique Paty, Les Pollueurs : luttes sociales et pollution industrielle, Paris, Seuil, 1980, 
véritablement exprimée qu'après une rupture, survenue dans l'après-Seconde guerre mondiale, du consensus autour des bienfaits de la société industrielle, à l'apogée de la mobilisation des défenseurs de l'environnement constatée au cours de la même période ${ }^{4}$. De même qu'une histoire de l'environnement n'aurait que peu de sens avant son institutionnalisation et son émergence dans le champ de la politique, celle des conflits environnementaux serait tout aussi dépourvue d'objet et exempte de troupes avant une date relativement récente. À quelques rares exceptions près, les publics étaient réputés ne pas protester (ou alors très peu), tout occupés qu'ils étaient à assurer pour eux-mêmes des conditions élémentaires d'existence. Il n'était pas alors question d'environnement, mais d'une adhésion au programme de rationalisation des usages de la nature par les sciences et les techniques ; bref, il s'agissait d'être parties prenantes du progrès industriel en marche.

\section{Du changement technique au changement climatique}

La société dite postindustrielle, la société du risque et de ses incertitudes, le traumatisme de la désindustrialisation et les conséquences des activités industrielles passées auront ainsi profondément transformé, à partir de cette période, les rapports qu'entretiennent publics, citoyens, salariés des usines et consommateurs avec l'industrie et ses débordements, mais aussi avec les experts et les autorités administratives en charge de la gestion sinon de l'environnement, tout au moins des intérêts contradictoires s'exprimant autour de la ressource naturelle, des territoires et de la santé.

Indéniablement, le contraste est saisissant entre l'exaltation d'une industrie triomphante, au début du siècle dernier, et sa stigmatisation, quelques décennies plus tard. Les rejets industriels auraient-ils suscité un rejet de l'industrie par l'opinion? La saturation des milieux naturels par les produits de l'activité industrielle et par les déchets non organiques, l'écologie scientifique et le militantisme pour la protection de la nature, ainsi que la sédimentation, dans les esprits, des catastrophes industrielles auront alors favorisé la mobilisation collective. Ainsi, l'évidence environnementale s'intégrerait parfaitement dans un grand récit sur la modernité, sur l'évolution au $\mathrm{XX}^{\mathrm{e}}$ siècle des sensibilités et sur l'histoire des rapports des publics à la nature, ponctuée par des accidents, des pollutions à grande échelle et des intoxications massives.

L'extension du domaine de l'environnement à tous les champs de l'activité humaine et l'injonction du développement durable invitent assurément à adopter cette vision d'un présent désormais responsable. À l'opposé, l'inconscience environnementale caractériserait les périodes antérieures. Elle aurait traversé l'époque de l'industrialisation tant parmi les populations que chez les élites et les décideurs. La contestation aurait été, en somme,

373 p. ; Philippe Le Prestre, « France's Administration of its Environment », International Review of Administrative Sciences, 47, 1980, pp. 42-50.

${ }^{4}$ Pour un modèle du genre : André Gortz, Écologie et politique, Paris, Seuil, 1978, 245 p. 
marginale, inaudible, sans justification légitime, soit du fait de l'ignorance des conséquences des impacts de l'industrie sur les milieux et la santé, soit parce que l'évidence de leur caractère inéluctable et nécessaire était largement partagée.

Mais le fait de s'en tenir à cette vision d'une conscience collective surgissant des ténèbres industrielles n'aide cependant pas à concevoir le poids du passé sur les attitudes et les comportements dans un présent plus immédiat. Par exemple, cette vision ne permet pas de clarifier certains paradoxes, dont, entre autres, celui qui fait apparaître sur le temps plus ou moins long des décalages chronologiques entre, d'une part, l'histoire des savoirs et des innovations techniques et, d'autre part, l'histoire des contestations environnementales et des mobilisations collectives et de celle de l'intervention publique (qui est aussi l'histoire de la législation). Le silence des riverains baignant dans les pollutions, à une certaine époque, contraste avec la virulence de leurs protestations qui s'expriment au moment même où la pollution en question n'existe pratiquement plus, voire plus du tout. Une lecture trop rapide pourrait alors nous conduire à devoir conclure à l'irrationalité des contestataires et à la disqualification de leur prise de parole dans l'espace public. C'est que les termes du conflit environnemental ne se limitent ni à la seule qualification experte de la pollution ni à un lien unidirectionnel entre une émission de toxiques et leur réception par des plaignants potentiels. Les enjeux sont plus complexes, dans lesquels la réalité biophysique des interactions entre des molécules et des organismes vivants, à l'évidence essentielle, demeure néanmoins insuffisante pour saisir un processus de nature profondément sociale. C'est précisément en cela qu'une interprétation des conflits locaux du passé à partir de la notion de débordement permet une restitution plus appropriée de leur histoire.

\section{Qu'est-ce qu'un conflit environnemental ?}

Le conflit environnemental doit-il être considéré comme un phénomène propre à la période la plus récente ? Une histoire, même sommaire, des pollutions industrielles et de leurs impacts montrera pourtant que celles-ci n'ont jamais été ignorées ni par les riverains ou par les ouvriers concernés ni par les autorités soucieuses de la santé, de l'hygiène et du maintien de l'ordre public ${ }^{5}$. Cette histoire implique toutefois de distinguer plusieurs niveaux d'analyse, d'échelles de temps et d'espace, de préciser les notions et les concepts permettant d'interpréter et de restituer tant l'histoire du temps présent que celle, plus ancienne, des pollutions et des conflits générés. Un de

\footnotetext{
${ }^{5}$ Outre les références déjà citées : Gérard Jorland, Une société à soigner. Hygiène et salubrité publiques en France au XIXe siècle, Paris, Gallimard, 2010, 361 p.; Sabine Barles, L'invention des déchets urbains : France, 1790-1970, Seyssel, Editions Champ Vallon, 2005, 297 p., et La ville délétère : médecins et ingénieurs dans l'espace urbain, XVIII ${ }^{e}$-XIX siècles, 1999, 373 p. ; André Guillerme, Anne-Cécile Lefort, Gérard Jigaudon, Dangereux, insalubres et incommodes : paysages industriels en banlieue parisienne, $X I X^{e}-X X^{e}$ siècles, Seyssel, Editions Champ Vallon, 2004, 343 p. ; André Guillerme, La naissance de l'industrie à Paris : entre sueurs et vapeurs, 1780-1830, Seyssel, Editions Champ Vallon, 2007, 432 p. ; Jérome Fromageau, La police de la pollution à Paris de 1666 à 1789, ANRT, 1989, 976 p.
} 
ses intérêts est qu'elle permet de saisir la nature des confrontations entre des enjeux contradictoires. En réduisant la focale, et en observant ainsi ce qui se joue au niveau le plus local, en s'éloignant du grand récit de la prise de conscience écologiste qui aurait permis la contestation, il devient possible de percevoir la pluralité des motifs et des rapports des acteurs à la ressource, aux territoires, à l'espace et au paysage, aux milieux. Cette démarche commande toutefois de préciser la notion de conflit environnemental au motif de débordements industriels.

Le conflit environnemental peut être d'abord regardé comme la manifestation locale de tensions entre des acteurs sociaux dont le motif est la confrontation des fonctions attribuées à un environnement donné. Ce dernier est cet espace reçu en partage dont il faut en permanence négocier les usages spécifiques entre des acteurs ayant des intérêts contradictoires. Cette confrontation des intérêts et la domination de certains d'entre eux sont alors susceptibles de générer une crise et l'émergence d'un conflit environnemental. Il y a donc conflit quand les fonctions attribuées à un environnement donné sont contrariées. Si elles le sont, c'est parce qu'elles portent atteinte à l'intégrité d'individus ou à celle de catégories de populations (d'êtres vivants, en particulier) ou à des écosystèmes dont les dynamiques d'équilibre sont menacées. Classiquement, ces intérêts sont contradictoires non seulement parce qu'ils contredisent l'usage et l'appropriation de ressources, d'espaces et de territoires, mais aussi parce qu'ils portent atteinte à des valeurs, à des identités, à des principes, à des rapports à la nature projetés sur cet environnement.

\section{L'historicisation du conflit environnemental}

Cette façon d'observer le conflit environnemental n'est pas spécifique à l'histoire du temps présent. Elle s'avère tout aussi pertinente pour interpréter les conflits du passé. Le conflit environnemental est lui-même un outil permettant de restituer l'histoire de ces confrontations entre des intérêts dont le motif est l'altération des milieux, l'usage de la ressource, le paysage, la santé, le territoire, le risque non consenti.

Le conflit environnemental n'a cependant pas été de toute éternité une évidence. Il faut historiciser cette idée d'un conflit dont l'environnement serait l'enjeu. Il faut pouvoir situer le sens de cette expression trop actuelle de «conflit environnemental», ses modalités d'existence dans le passé. Autrement dit, il s'agit non pas de faire dire aux acteurs du passé qu'ils étaient impliqués dans un conflit environnemental, mais d'interpréter et de restituer, à partir de l'histoire de ce qui nous paraît aujourd'hui avoir relevé à l'époque de l'ordre du conflit environnemental. Enfin, le conflit environnemental ne doit pas être conceptualisé comme un conflit portant sur un problème prédéfini et sans équivoque, avec des acteurs dont les points de vue et les intérêts seraient en concurrence ; il doit plutôt être vu comme une négociation complexe et permanente visant à assigner une définition et une signification au problème environnemental lui-même. 


\section{Quel environnement ?}

De la même façon, il faut éviter l'anachronisme qui consisterait à mobiliser une conception actuelle de l'environnement pour l'appliquer à des situations du passé, qui n'auraient rien à voir avec ce que vivaient à l'époque les protagonistes. L'environnement est donc compris ici d'une façon extensive et compréhensive intégrant tout ce que peuvent comporter en fait de nature et de culture des espaces ayant été appropriés par des populations, des partisans et des opposants se disputant les fonctions et le statut de cet environnement.

L'environnement invoqué concerne dès lors autant des milieux biophysiques, des ressources naturelles, des paysages, des situations sanitaires, des espaces et des territoires, des écosystèmes dotés de leurs dynamiques propres que des structures humaines faites de nature et d'organisations politique et sociale. Autrement dit, le terme «environnement» ne réfère ni à un état de nature (auquel une valeur intrinsèque serait nécessairement attribuée) ni à cette catégorie, spécifique, de l'action ou de la pensée politique ou encore seulement de l'intervention publique, mais, plus simplement, à l'ensemble de ces choses environnantes faites de nature et de culture, inscrites dans des histoires et constituant des territoires, dont les statuts et les qualifications font l'objet à un instant donné d'une renégociation, d'une réaffirmation à travers le conflit.

\section{Une histoire pragmatique du conflit environnemental}

Au demeurant, envisager une histoire de la conflictualité environnementale suppose de considérer la polysémie du terme "environnement ». Celle-ci impose de situer son improbable définition au centre d'une négociation permanente portant sur les frontières entre des espaces publics et des espaces privés, entre culture et nature, entre des logiques techniques et les mondes du vivant, ou entre des systèmes techniques et des réalités vécues.

Cette façon d'appréhender l'environnement comme le motif d'un conflit oblige ainsi à combiner plusieurs registres d'un réel qui est toujours pluriel. La démarche interdisciplinaire s'impose pour saisir tout ce qui compose ce conflit et ce qui le fait advenir à la fois comme une réalité constituée par des phénomènes naturels et sociaux, comme une réalité territoriale et comme une réalité inscrite dans un contexte historique.

Il faut tenir compte de trois composantes irréductibles, trois registres de considérations dont aucun ne peut jamais être totalement ignoré, même s'ils peuvent, bien entendu, devoir être isolés pour les besoins de l'enquête historique :

- une composante géographique et spatiale, qui est celle des territoires au travers desquels s'exprime le conflit environnemental, 
- une composante historique et temporelle, qui est celle des histoires dans lesquelles s'inscrivent les termes du conflit environnemental,

- enfin, une composante hybride, c'est-à-dire celle de l'articulation du réel tangible et du social qui permet de faire advenir le conflit environnemental dans des ordres de réalités plurielles et humaines (voir la figure 1).

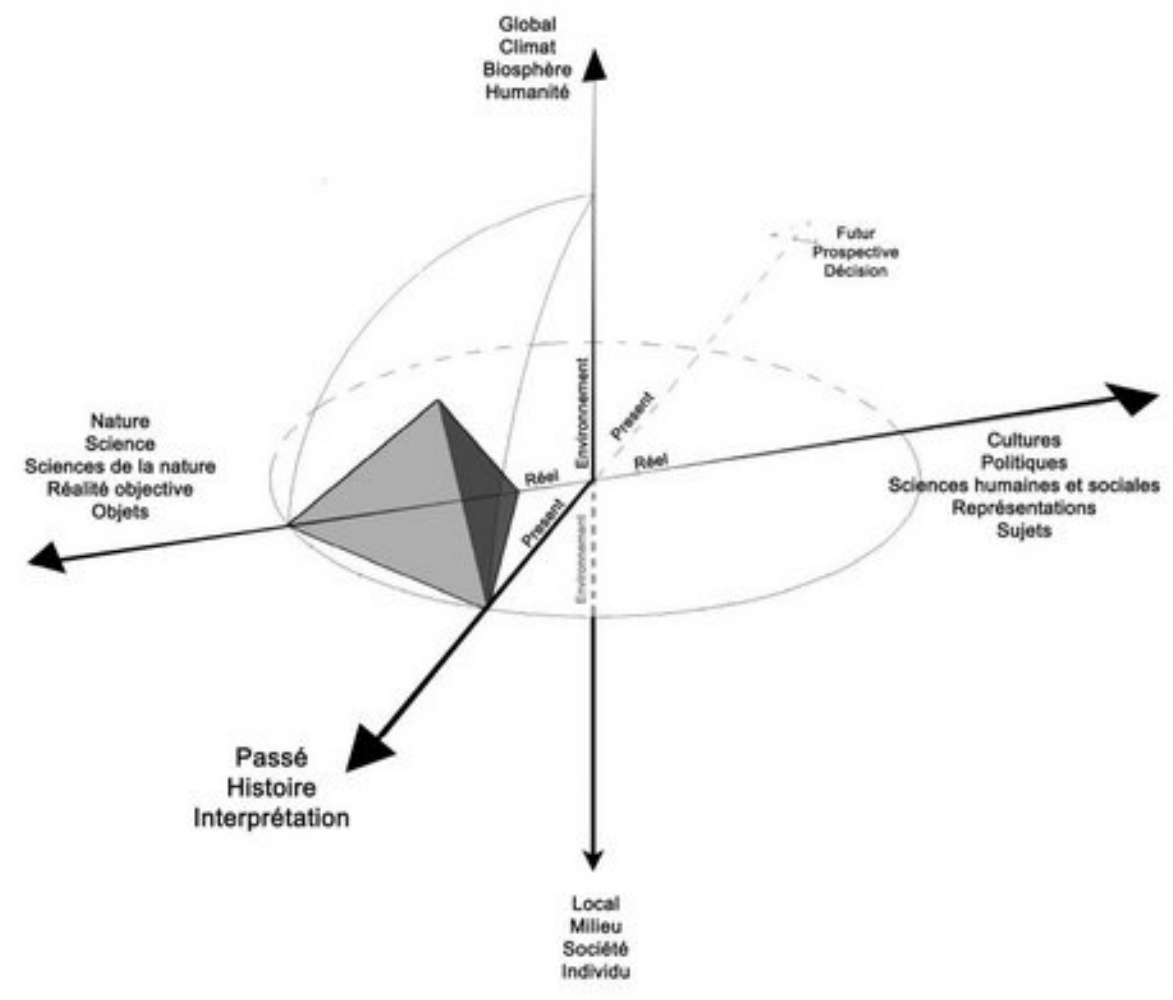

\section{Un motif récurrent de conflit environnemental : le débordement}

Les origines d'un conflit environnemental peuvent être des plus diverses, mais toutes auront pour motif la remise en cause soit d'une situation environnementale désormais contestée, soit d'une évolution du fait d'un facteur menaçant un état d'équilibre antérieur. Nous nous intéresserons ici à un motif particulier de conflit, le débordement industriel, c'est-à-dire ce qui, provenant d'un dispositif de production, investit un environnement donné et suscite la protestation de ceux qui y vivent.

Cependant, pourquoi référerons-nous au terme « débordement », plutôt qu'à ceux de «pollution » ou de « nuisance », assurément plus clairs ? D'abord, parce que ce terme de débordement implique deux conséquences qui paraissent moins évidentes lorsque l'on ne considère que les seuls termes, plus restrictifs, de pollution et de nuisance :

- Le motif du conflit est ainsi attribué, sur le mode de la responsabilité, à un dispositif de production d'où émane ce quelque chose qui génère 
la contestation des populations alentours. Le fait de prendre en considération des débordements permet de n'exclure aucun des motifs possibles du conflit. Le terme de débordement a été, de fait, choisi en raison de sa souplesse et de sa richesse : il permet, en effet, d'inclure l'ensemble de ce qui est susceptible de perturber une situation environnementale selon les trois registres irréductibles mentionnés précédemment : l'histoire, le territoire et le caractère nécessairement hybride des motifs du conflit.

- L'ambigüité et la multitude des phénomènes que le terme « débordement » englobe, renvoient non seulement à une histoire des résultats, des faits ou des conséquences tangibles, mais aussi, et surtout, aux processus par lesquels ces derniers adviennent. Le terme « débordement» oblige ainsi à devoir restituer les traductions, qualifications et justifications de ce qui, outrepassant les limites ou les frontières d'un dispositif de production donné, entre en conflit avec l'environnement de celui-ci. Restituer l'histoire des débordements à l'origine d'un conflit local, cela revient à restituer les processus par lesquels les modalités d'insertion d'un dispositif de production et de ses extensions sont devenues des débordements, c'est-à-dire comment elles ont pu devenir le motif d'un conflit. Le conflit devient le siège d'une négociation entre des agents, des parties prenantes, des responsables, des victimes, des populations lésées, des porteurs d'enjeux, bref, le siège d'une négociation entre des acteurs concernés ou affectés qui tentent de faire exister ou disparaître les débordements ainsi désignés.

Le débordement semble donc, de ce point de vue, une lecture bien plus pertinente pour une tentative de restitution de l'histoire des conflits locaux dont le motif est l'insertion d'un dispositif de production dans l'environnement. Ramener à des débordements les origines et les justifications de ces conflits, considérer ces débordements comme leurs motifs, cela suppose dès lors que l'on établisse une histoire des modes d'existence et d'extension du dispositif de production dans son environnement.

\section{Quelques exemples de débordements industriels (au XIX ${ }^{\mathrm{e}}$ siècle)}

L'expression « débordement industriel » est par ailleurs une expression que l'on rencontre assez fréquemment au $\mathrm{XIX}^{\mathrm{e}}$ siècle. Elle désigne des situations des plus diverses, mais qui ont toutes pour corollaire l'idée d'une emprise de l'industrie sur la société, avec le soupçon sous-jacent de sa corruption. Cette notion est mobilisée pour dénoncer la menace que la production industrielle fait peser sur des fabrications traditionnelles. Ce sont, par exemple, les termes du débat autour de la production industrielle d'eaux minérales qualifiées d'artificielles, auxquelles sont attribuées des vertus douteuses; celles-ci menacent de remplacer les eaux minérales naturelles, aux vertus avérées. C'est encore la production industrielle de spécialités pharmaceutiques fabriquées d'avance en grandes quantités et vendues au public; celles-ci 
menacent de remplacer les préparations galéniques du pharmacien prescrites par le médecin. L'expression de débordement industriel est ainsi mobilisée dans cette lutte entre l'ancien et le moderne, par exemple quand il est question d'une concurrence entre le développement agricole et l'expansion de l'industrie ou, encore, dans un tout autre registre, quand il est question (chez Sainte-Beuve) du déferlement de la littérature industrielle de médiocre qualité, qui menace la production des œuvres nobles et véritables. Mais les termes «débordement industriel» sont bien plus encore utilisés non seulement pour pointer plus particulièrement l'expansion territoriale de l'industrie, son accaparement des ressources naturelles, les dégradations qu'elle inflige aux milieux naturels, mais aussi pour signaler ce qui devient inacceptable pour des populations alentour quand il s'agit de dénoncer les effets d'une activité industrielle donnée sur le milieu et la santé publique. L'expression et l'idée se retrouvent ainsi chez les médecins et les hygiénistes, durant la seconde moitié du XIX ${ }^{\mathrm{e}}$ siècle, dans la littérature spécialisée, comme la Revue d'hygiène et de police sanitaire, où les débordements industriels apparaissent à maintes reprises. Voilà des exemples parmi d'autres où l'industrie est décrite et caractérisée par ses débordements, c'est-à-dire ses excès, le déversement de ses produits et l'imposition de ses logiques qui menacent de corrompre la société.

Pourquoi ce rappel ? Pour dire que le débordement industriel est une réalité ancrée dans les esprits de l'époque et qu'il n'est donc pas absurde de convoquer cette notion comme outil possible d'interprétation de l'histoire des conflits environnementaux locaux. Le débordement industriel, on le voit dès le $\mathrm{XIX}^{\mathrm{e}}$ siècle, ne va pas de soi. L'extension de l'industrie est susceptible, sous certaines de ses formes, de poser des problèmes au premier rang desquels on trouve, évidemment, les questions liées aux pollutions industrielles et à l'environnement (l'environnement de l'usine, de l'atelier ou de l'entreprise, mais aussi celui dans lequel vivent les populations riveraines).

\section{Que recouvre le terme de « débordement »?}

La notion de débordement offre, comme on l'a vu, la perspective d'inclure dans le champ de la contestation environnementale toutes les manifestations par lesquelles les externalités d'une activité industrielle peuvent être amenées à se traduire par des interactions contestables (et contestées) en lien avec l'environnement. Le mot contient le problème : ce qui déborde est soupçonné d'illégitimité, évoque l'abusif, ce qui demande à être contenu, contrôlé, surveillé, voire stoppé, sous peine de catastrophe. Le débordement suggère l'incommode, le désagréable, les mauvaises odeurs et l'impropre. Mais il n'est pas toujours clairement identifié ; son statut reste bien souvent ambigu.

Toutefois, le débordement ne relève pas nécessairement du tangible ; il peut aussi être immatériel. Ainsi, il peut concerner tout ce qui est susceptible de générer ou d'entretenir un conflit entre un dispositif de production et son environnement : 
- débordements physiques, directement décelables par les sens : odeurs, miasmes, fumées, vapeurs, bruits, trépidations, poussières, vue intolérable ou immorale, effluents liquides déversés dans les cours d'eau ou les espaces publics (ce sont les nuisances et les pollutions proprement dites),

- des débordements potentiels et redoutés : des risques (d'explosion, de catastrophe, d'incendie, d'épidémie...) et des dangers (supposés, fantasmés ou avérés),

- des débordements invisibles, inconnus ou non perceptibles (vecteurs de contamination, de radiations, d'émanation de toxiques incolores et inodores),

- des débordements d'ordre économique et social : concurrence pour l'usage de la ressource, effet de la présence d'une entreprise sur le comportement des propriétaires fonciers (spéculation immobilière),

- des débordements différés dans le temps: effets cumulatifs de polluants qui ne révèlent leurs conséquences qu'après une période plus ou moins longue, ou qui ne seront connus qu'une fois acquis les connaissances ou les instruments permettant de les déceler et de les évaluer,

- des débordements insidieux: emprise d'une entreprise sur les populations alentour en matière de domination et de contraintes; effets sur le comportement des populations riveraines et influence sur l'organisation de la vie locale,

- des débordements induits : les inconvénients que peut générer le fait même de l'existence d'une activité, par exemple en raison de l'attraction qu'elle exerce ; les inconvénients que cela peut être amené à représenter pour les populations alentours (une circulation problématique aux abords du site de production, des formes contestées de développement urbain, une immigration ouvrière), avec des troubles connexes pour l'ordre public.

Une activité industrielle ne produit pas que des externalités négatives : en effet, toutes les interactions que nous avons mentionnées n'ont pas forcément un impact délétère (les modalités d'interaction entre un dispositif et son environnement ne sont pas inéluctablement conflictuelles).

L'enjeu est donc d'identifier ce qui est en passe de devenir un débordement constituant le motif d'un conflit. Il est de comprendre de quelle manière et pour quelle raison un débordement donné a été perçu et vécu comme tel, et pourquoi il a été jugé nécessaire de le combattre, ou au contraire de feindre l'ignorance, ou encore d'en accepter les conséquences.

\section{Le débordement : un dispositif centré et distribué}

L'identification des débordements ne peut être dissociée de la question de leur origine, de la source même de leur production. Que faut-il entendre par « activité débordante»? Quelle est cette activité productrice de ce « quelque 
chose » qui, outrepassant les limites de l'usine ou de l'atelier, est considéré comme un débordement affectant l'environnement?

Si l'on réduit ladite activité à sa dimension géophysique, il est facile de se figurer un dispositif de production occupant un espace, de voir un atelier ou un champ d'exploitation, de saisir dans leur matérialité des flux entrants et sortants, des substances émanant de leur lieu de production. D'un côté, un « dedans » produit des matières dont tout ou partie est rejeté au « dehors ». De l'autre, un milieu s'imprègne, s'altère ou se transforme de façon plus ou moins perceptible du fait de ces débordements. L'affaire est claire et chacun peut sans aucune difficulté mobiliser un exemple. Le dispositif de production exerce, dans ce cas, une activité, occupe un espace dont les limites sont fixées par ses droits de propriété. Ses frontières se matérialisent par des lignes inscrites sur un plan cadastral. Un «dedans » et un « dehors » clairement délimités permettent de désigner ce qui transgresse les frontières et affecte les fonctions de territoires partagés, c'est-à-dire n'appartenant pas exclusivement à l'entreprise.

Rien ne serait cependant plus illusoire que de s'en tenir à ce qui sépare un dispositif du milieu dans lequel il s'insère et qu'il contribue à façonner. On ne peut se contenter de considérer un espace duquel s'échapperait ce " quelque chose », qui, en entrant en interaction avec l'environnement immédiat, susciterait la contestation. Cela reviendrait à considérer l'activité de production comme étant une entité rigide, statique, dépourvue de dynamique propre et close, une entité dont l'intérieur et le dedans se distingueraient nettement de l'extérieur, de ce qui est en dehors d'elle. Les débordements ignorent tout simplement cette séparation nette entre des territoires clairement identifiés et fixés une bonne fois pour toutes. Des territoires aux statuts négociés qui s'imbriquent, s'intriquent les uns les autres, se chevauchent et participent à la constitution d'un dispositif de production fait de l'activité industrielle et de ses débordements. Il s'agit d'un dispositif précaire, car chaque territoire est revendiqué pour sa possession et son usage légitime, est négocié pour maintenir son intégrité et défendre des modes de jouissance face à la menace de leur contestation. A tout moment, un nouvel acteur ou un nouvel agent, un élément, un enjeu ou un intérêt particulier peut le remettre en cause.

Poser ainsi le problème, c'est insister sur la détermination des limites du champ d'interaction, de l'espace d'implication et d'influence, c'est-à-dire insister sur les frontières et les limites au-delà desquelles il y a « débordement », et donc contestation territoriale.

Là réside une difficulté majeure, celle de saisir ce qu'est, au juste, l'activité débordante, ce par quoi elle s'incarne. Cette dernière n'est pas une, elle est une multitude d'entités opérationnelles plus ou moins autonomes, d'incarnations dans des collectifs de travail qui sont eux-mêmes insérés dans des réseaux étroits de contraintes, de partenaires, de collaborateurs, de clients, de fournisseurs, d'usagers, de salariés. En somme, un dispositif de production est cette entité sociale, économique, politique et culturelle, à la fois 
« centrée » et « distribuée », de laquelle débordent ces choses matérielles ou intangibles qui sont à l'origine des conflits territoriaux.

- une entité centrée, parce qu'il est toujours possible de s'en tenir à une délimitation stricte de son espace par ses limites (physiques ou fixées par le législateur). Dans ce cas, une activité constitue un dispositif dédié à la production dont les émanations suscitent la contestation des populations alentour. Clairement délimitée, par exemple, par les murs de la fabrique, l'activité centrée est au cœur du champ de bataille ; elle est cernée par les protestations, les habitants, les propriétaires fonciers, les riverains, les associations et les groupes concernés.

- une entité distribuée, parce que ce dispositif est toujours plus ou moins localement disséminé dans la vie et le tissu social, dispersé dans des intérêts individuels et collectifs, éparpillé parmi les acteurs de la vie locale et du tissu urbain. Il faut donc rendre compte de ses modes d'existence non seulement dans des espaces publics, mais autant que possible, également dans des espaces privés. Le dispositif de production participe de l'existence de réseaux d'affaires, techniques, financiers et commerciaux, et c'est sa capacité à s'interconnecter et à multiplier les points de contact qui lui permet d'assurer son développement, de se déployer dans l'espace, bref, de déborder le cadre initial de ses activités pour les inscrire dans la durée.

Cependant, le propos n'est pas de multiplier, pour les confondre, des espaces de toute nature, mais de considérer les interfaces territoriales qu'un dispositif centré et distribué se doit de négocier dans la confrontation et le conflit environnemental. Il n'est pas non plus de dire qu'il n'existerait pas de frontière véritable entre un dispositif et l'espace qui l'entoure (le mur d'enceinte d'une usine matérialise indéniablement une séparation pertinente entre un dedans et un dehors), mais bien de dire que l'on ne peut ignorer des territoires d'une nature plus équivoque et ambigüe. Quand bien même peut paraître simple le cas d'une émanation toxique traversant les limites matérielles d'un espace supposé clos, un dispositif de production manifeste son existence parmi les populations alentour autrement que par la seule pollution que génère son activité. Elle négocie toujours sa présence en imposant une multitude de territoires dont les échelles, la nature, les significations et les conséquences varient en permanence en fonction de leur reconnaissance par les acteurs locaux.

Dispositif technique et entité sociale, à la fois physique, institutionnelle, juridique, économique, politique et culturelle, l'activité structure de fait le milieu sociotechnique et bio-social dans lequel elle se distribue et que la « mise en crise » oblige à renégocier. C'est l'entité « dispositif » qui demeure à la fois source, responsable et partie prenante, l'acteur principal au nom duquel s'exerce l'activité aux origines des débordements dans la cité. C'est avec ce dispositif, ses représentants et ses défenseurs d'intérêts particuliers que se négocient l'assimilation, l'ignorance, l'effacement ou la disparition des débordements incriminés. La démarche implique donc de délimiter les territoires de l'entreprise au-delà desquels il y a «débordement», ses extensions, ses modes de distribution dans l'environnement. Elle consiste, 
dans ce cas, à désigner ce par quoi un dispositif s'incarne, à délimiter la frontière matérielle au-delà de laquelle son domaine exclusif n'est plus, mais devient celui, plus ou moins partagé, de populations alentour, du domaine public, d'autres entreprises ou d'activités concurrentes.

Cependant, la désignation de ce qui déborde nécessitera toujours de clarifier non seulement ce qui sépare, distingue, fait limite, détermine les normes, les seuils, les enceintes explicites ou tacites, mais aussi ce qui permet, dans tous les cas, de saisir le sens même des « débordements » à l'origine d'un conflit. Le dispositif n'est donc pas seulement le moyen technique de la réalisation de la production, il est aussi une institution, l'instrument d'une emprise et de la domination, un acteur local avec lequel il est inévitable de négocier des règles et des manières de concilier des intérêts contradictoires.

\section{Les territoires du débordement}

Les débordements locaux sont une des variantes des motifs à l'origine de conflits d'implantation et d'usage. L'examen de ce type de conflits a d'ailleurs été, ces dernières années, l'objet d'un certain engouement de la part des pouvoirs publics, et conséquemment des chercheurs. Un des résultats rappelé dans la plupart de ces travaux est que les territoires ne sont jamais figés, que les groupes sociaux ne préexistent pas nécessairement aux actions collectives, mais que les modes de relations aux territoires se négocient en permanence, au travers de ces conflits constitutifs d'une transformation des rapports à l'espace, de la territorialisation des débordements et de leurs conséquences.

Un dispositif de production est toujours territorialisé. Un dispositif exerce toujours une emprise territoriale par son implantation, par les circulations qu'impliquent ses flux de ressources, le recrutement et le traitement de sa main-d'œuvre, ses annexes, ses effluents et les risques qu'il génère pour les populations alentours et l'environnement.

Ces territoires sont des espaces géographiques, physiques et socialement construits par le fait même d'une activité. Ils ont une fonction essentielle, celle d'aménager les conditions optimales de la réalisation de la production. Le territoire est donc en soi un enjeu primordial. Tout dispositif cherche à s'en assurer la maîtrise, la reconnaissance, c'est-à-dire le droit, sinon le devoir, d'en user selon ses intérêts propres.

Une des conditions de sa pérennité et de son éventuelle expansion réside donc dans la production et la stabilisation de territoires que le dispositif de production est amené à s'approprier en permanence. Une industrie, un atelier, un champ d'exploitation ou une mine ne sont jamais posés sur une terre inhabitée, vierge de toute histoire, mais ils interagissent, en permanence, avec leur environnement ; ils s'ancrent dans des cultures locales et s'inscrivent dans un paysage. Un dispositif mobilise des ressources disponibles à proximité, transforme et met en circulation des matières, des individus, et 
génère des flux de marchandises. Par ses échanges permanents avec un extérieur, il transforme le milieu dans lequel il s'insère jusqu'à, parfois, le façonner dans son intégralité. Le dispositif territorial, par la multiplication des espaces nécessaires à l'exercice de son activité principale - la production -, est une organisation d'origine et de nature sociales, qui implique une relation entre territoire et pouvoir. Elle s'appuie sur la négociation plus ou moins explicite pour la reconnaissance de zones de compétence et de sphères d'influence, de droits sur des fractions de territoires proportionnels aux pouvoirs que le dispositif peut y exercer.

Le territoire, compris comme une portion d'espace géographique dont la délimitation et le contrôle visent à exercer un pouvoir sur une population et des ressources, doit être utilisé lui-même comme un outil d'analyse des conflits environnementaux. Autrement dit, une implantation industrielle est regardée comme ce dispositif de production dont la dynamique de survie et d'expansion exige une appropriation permanente de nouveaux territoires. Elle permet à l'activité débordante d'assurer ses besoins en espace et en ressources, c'est-à-dire de garantir l'assimilation de ses externalités.

Les débordements contestés sont dès lors distingués comme des extensions territoriales du dispositif de production qui leur donne naissance. L'objectif est d'identifier ces fragments de territoire en concurrence. Il est aussi de comprendre leurs modes de constitution, leurs stratifications et leurs intrications, invitant ainsi à examiner les débordements eux-mêmes en tant qu'enjeux territoriaux. Car les conflits du fait de débordements renvoient toujours, d'une façon ou d'une autre, à l'impératif d'une négociation pour la qualification, la justification et la légitimation des fonctions associées à ces territoires disputés par (et pour) l'activité à l'origine des débordements incriminés. C'est pourquoi la négociation est l'enjeu majeur de la construction de ces territoires du débordement, qui est fondée sur le besoin de création constante de ressources pour l'entreprise.

Le territoire du débordement ne se réfère pas seulement à une zone, à une superficie, à une forme, à des limites matérielles et à des contours physiques, il renvoie également à l'organisation d'un espace constitué de nature et de culture, de matières et de pouvoirs, d'objets et de sujets. Les débordements sont dès lors regardés comme des espaces nécessairement hybrides, où les fondements objectifs du territoire matérialisent des enjeux politiques et sociaux. La restitution par l'histoire d'un conflit environnemental doit dès lors privilégier l'articulation problématique de ces composantes territoriales physiques et naturelles avec des éléments de territoire socialement construits et disputés. Il faut, dans ce cas, s'attacher à définir le « territoire » de manière extensive. Cette définition impose d'investir les processus de territorialisation qui sous-tendent la constitution du territoire.

La notion de territoire à négocier lors d'une confrontation entre des fonctions territoriales contradictoires permet ainsi de pourvoir aux besoins de l'enquête historique. Elle sert d'outil d'interprétation des modalités d'insertion du dispositif de production dans un milieu local, elle permet d'examiner la multiplicité des interactions que ce dispositif instaure avec les populations 
alentour et l'environnement. La (re)qualification permanente de ses territoires autour de la ressource ou de l'usage commande à l'évidence non seulement d'explorer la variété des enjeux et de leurs confrontations, mais aussi de restituer les stratégies mises en œuvre pour garantir la légitimité de ces territoires et de leurs fonctions, que les parties prenantes leur attribuent et se disputent.

Dans un conflit environnemental où les rapports sociaux déterminent et structurent des espaces négociés, ces notions territoriales et leurs articulations problématiques font sens : elles renvoient à l'idée d'une stratification de territoires concurrents et à leur intrication par des liens d'interdépendance, d'interrelations, d'interactions, de tensions ou de contraintes. Ce sont ces liens que les conflits révèlent et qui se manifestent avec plus ou moins de violence et d'évidence. Une des fonctions du conflit est bien alors d'inscrire dans l'espace public la négociation de ces contrats explicites et tacites, discutés et imposés, qui ont abouti, un temps, à la justification de débordements désormais contestés. Le fait de restituer par l'histoire un conflit environnemental converge ainsi vers la restitution de ces négociations en vue d'une cohabitation, toujours précaire, entre des territoires aux fonctions contradictoires.

\section{La négociation par le conflit environnemental}

Il faut donc dire ce qui constitue ces lieux concrets, réels et symboliques, inhérents aux débordements et préciser tant les activités dénoncées que les processus concurrents d'appropriation de ces territoires dont les fonctions initiales sont contrariées par ce qui déborde. La démarche implique de saisir autant que possible les négociations en vue de l'attribution de ce statut de « débordement » aux matières réelles ou intangibles concernées. Ce sont ces négociations qu'il s'agit de restituer par l'histoire des conflits environnementaux, des négociations ayant pour siège les arènes publiques de débat, la cité administrative et politique, les enceintes judiciaires, les espaces de production de la norme, les domaines spécifiques de l'intervention publique, les lieux de la prise de décision et ceux de la science et de l'expertise.

L'intention est donc d'identifier le dispositif de production à l'origine des débordements et du conflit subséquent et de situer les territoires qu'ils constituent, de saisir la nature des enjeux dont ces territoires sont le siège, de dégager, pour chaque acteur, les termes de la négociation visant à qualifier les débordements et à déterminer leurs frontières, enfin d'aboutir à la restitution d'une interprétation plurielle de ces débordements dans la cité, ou plutôt dans les cités dans lesquelles ils sont traduits.

Les débordements industriels ne sont jamais univoques ; ils sont, toujours, protéiformes. Leur reconnaissance et leur qualification font l'objet de négociations dans ces cités comprises comme les lieux de leur traduction, pour les faire advenir dans des ordres de réalités biophysique, juridique, 
culturelle, politique, économique, etc. Il est évident que les termes de ces négociations au cours d'un conflit environnemental ne peuvent être tout à fait les mêmes selon que l'on observe ces négociations du point de vue de l'industriel dans la cité entrepreneuriale ou de celui du riverain dans la cité médiatique ou publique, par exemple. Dans tous les cas, demeure le défi de disposer des éléments pertinents pour une histoire interdisciplinaire des conflits auxquels ces confrontations territoriales donnent lieu. Il serait la restitution de ces négociations dans autant de cités que possible afin d'aboutir à la proposition d'une histoire pragmatique et compréhensive des conflits environnementaux locaux passés.

Michel Letté 